\title{
Diagnosis of acute myocardial infarction from sequential enzyme measurements obtained within 12 hours of admission to hospital
}

\author{
P O COLLINSON, E M RAMHAMADANY, S B ROSALKI, $\dagger$ J JOFFE, ${ }^{*}$ D H EVANS, \\ R S FINK, T W GREENWOOD, I M BAIRD*
}

From the Departments of Clinical Biochemistry and ${ }^{*}$ Medicine, West Middlesex University Hospital, Isleworth, and the †Department of Chemical Pathology and Human Metabolism, Royal Free Hospital, London

SUMMARY A prospective study was made of sequential changes in serum creatine kinase (CK) and CK-MB isoenzyme activity within the 12 hours following admission to the coronary care unit on 65 patients with recent chest pain. CK determinations were performed in the laboratory or in the coronary care unit using a dry reagent strip analyser. Slope values for $\log$ CK/hour and $\log$ CK-MB/ hour were calculated, used to confirm or exclude the diagnosis of myocardial infarction, and compared with diagnosis by conventional means. Compared with retrospective diagnosis using all available information, the CK slope had a sensitivity of $100 \%$ and a specificity of $94 \%$. This compared with a sensitivity of $94 \%$ and specificity of $90 \%$ for diagnosis using upper reference limits alone. Determination of CK slope permits very rapid and accurate biochemical confirmation or exclusion of myocardial infarction and the possibility of performing the measurements on the coronary care unit. It additionally offers the prospect of major cost savings resulting from earli discharge or transfer from the coronary care unit.

The exclusion of acute myocardial infarction remains a problem in the patient presenting with acute chest pain. The electrocardiogram (ECG) is commonly used for diagnosis, but ECG changes may be equivocal or obscured by dysrythmias or bundle branch block. An estimated $56 \%$ of diagnoses of myocardial infarction require confirmation by some other investigation, typically a "cardiac enzyme" profile.' The conventional strategy of measurement of "cardiac enzymes" on consecutive days following admission, however, will frequently result in unnecessary hospital stay before diagnosis is confirmed or excluded, because $53-74 \%$ of the admissions to the coronary care unit may not have acute myocardial infarction. ${ }^{2-4}$

We have previously shown that sequential changes in the logarithm of creatine kinase (CK) activity and its $\mathrm{MB}$ isoenzyme (CK-MB) activity in the 12 hours following admission (CK and CK-MB slope) can be used to confirm or exclude the diagnosis of myocardial infarction in patients with acute chest pain. ${ }^{5}$ We report on the results of a more extended prospective study of

Accepted for publication 12 June 1989 this technique and its use in "near-patient" testing for the definitive diagnosis of myocardial infarction in coronary care unit.

\section{Material and methods}

Blood samples were obtained on admission and four, eight, and 12 hours later from consecutive patients admitted to the coronary care unit over four months. Sera were separated and stored frozen at $-20^{\circ} \mathrm{C}$ until analysed for total CK and CK-MB (CK-2) activity. All samples were analysed within one week of collection.

All patients were assessed by clinical examination and serial electrocardiography. The cardiac enzyme N protocol in use in the laboratory (measurement of aspartate transaminase (AST) and hydroxybutyrate $N$ dehydrogenase (HBD) for three consecutive days after $\mathbb{N}$ admission) was also followed. This is referred to as the "standard" enzymes. Full clinical details were 0 recorded prospectively on each patient by proforma.

AST and HBD were measured at $30^{\circ} \mathrm{C}$ by opti- $\stackrel{\oplus}{+}$ mised methods ${ }^{6}$ with commercially supplied reagents 
(Merckotest GOT(ASAT), Merckotest HBDH, BDH Diagnostics, Poole, Dorset). Total CK was measured at $30^{\circ} \mathrm{C}$ by an optimised method ${ }^{6}$ and CK-MB at $30^{\circ} \mathrm{C}$ by immunoinhibition, both using commercially supplied reagents (CK NAC opt., CK-MB NAC opt., BCL, Lewes, Sussex). All determinations were made using a centrifugal analyser (Encore, Baker Instruments, Windsor, Berkshire). CK was also measured using a dry strip method on the Ames Seralyser (Ames Laboratories, Stoke Poges) on the coronary care unit by a member of the staff. This analyser had been previously assessed for precision and accuracy over the analytical range used. ${ }^{\text {? }}$

All clinical records, electrocardiograms, and changes in the standard enzymes were retrospectively reviewed and a final diagnostic classification produced. Results of echocardiography, gated pool studies of technetium m99 multiple uptake, stress electrocardiography, and 12 lead ECG from previous hospital attendance and at follow up were used, where available. Infarction was judged to have occurred in patients with a history of chest pain accompanied by the development of $Q$ waves, or ischaemic ECG changes and sudden death within 72 hours of admission. S-T and T wave changes were taken to indicate infarction if there were typical changes in "standard" cardiac enzymes or evidence from cardiac imaging. Persistent residual S-T depression or $\mathrm{T}$ wave changes without changes in "standard" enzymes were classified as ischaemia ?infarction. Angina was diagnosed in patients with clinical features suggestive of ischaemic heart disease but without "standard" enzyme changes, transient S-T changes, or a positive stress ECG. Crescendo angina was diagnosed when ECG and "standard" enzymes intially indicated ischaemia alone in a patient with persistent pain but changes typical of myocardial infarction occurred 48 hours after admission. Non-ischaemic chest pain was diagnosed when angina could be excluded by a definite alternative source of the chest pain or an atypical history with a negative stress ECG. Information from changes in CK and CK-MB in the serial samples was not used in this classification.

In patients presenting with chest pain of onset within the previous 12 hours CK and CK-MB slope were calculated, as previously described, ${ }^{5}$ from CK and CK-MB values obtained from the sequential serum samples. Classification into infarct and non-infarct groups was performed using the $\mathrm{CK}$ and CK-MB slope values obtained from the previous study (log enzyme change with time, CK or CK-MB slope is independent of assay temperature for optimised methods). Patients with slope values exceeding 0.015 were classified as slope positive, indicative of recent myocardial infarction.

Enzyme diagnosis of infarction in these serial samples using conventional upper reference limits was also examined. These upper reference limits were: $\mathbf{C K}$ $120 \mathrm{U} / \mathrm{l}, \mathrm{CK}-\mathrm{MB} 12 \mathrm{U} / \mathrm{l}$, and greater than $5 \%$ of the total activity.

\section{Results}

Sixty five patients presented with symptoms of less than 12 hours' duration (53 men, 12 women), age range $40-86$ years, median 63 years. Using the criteria defined above, 34 patients were considered to have sustained myocardial infarction, five to have had ischaemia ?infarction, one to have had crescendo angina, 15 to have had angina and 10 to have had nonischaemic chest pain.

\section{DIAGNOSIS BY CK AND CK-MB SLOPE}

(LABORATORY DETERMINATIONS)

Results comparing the final diagnosis with classification using CK slope are shown in fig 1. CK slope

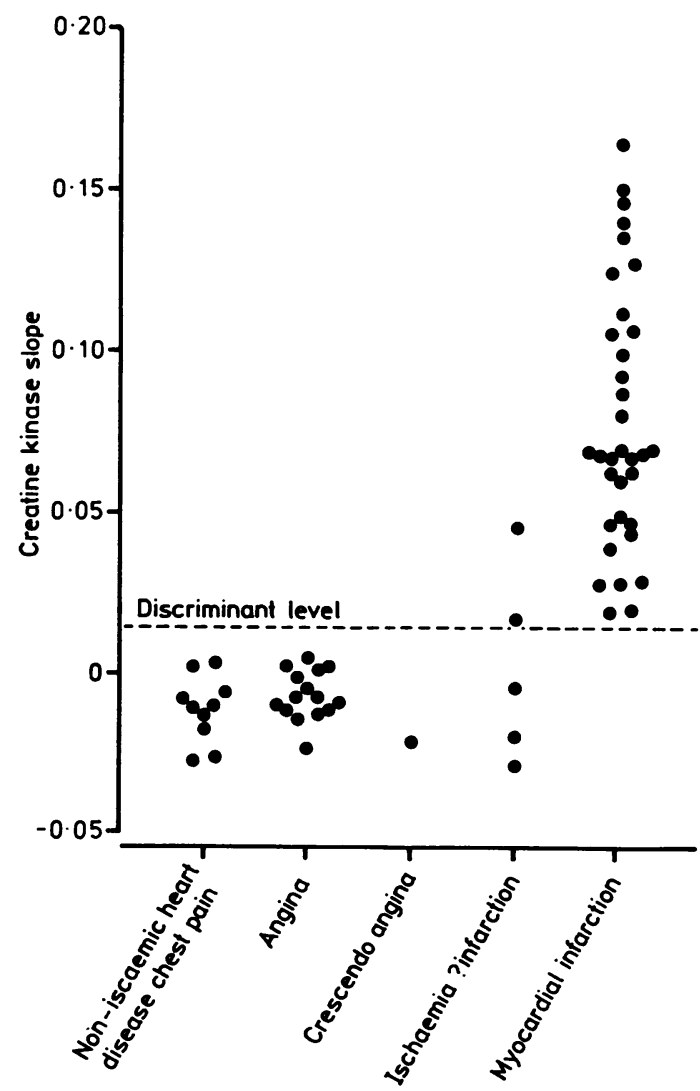

Fig 1 Change in log CK per hour (laboratory determinations) using final diagnostic classification in patients with chest pain of 12 hours or less. 


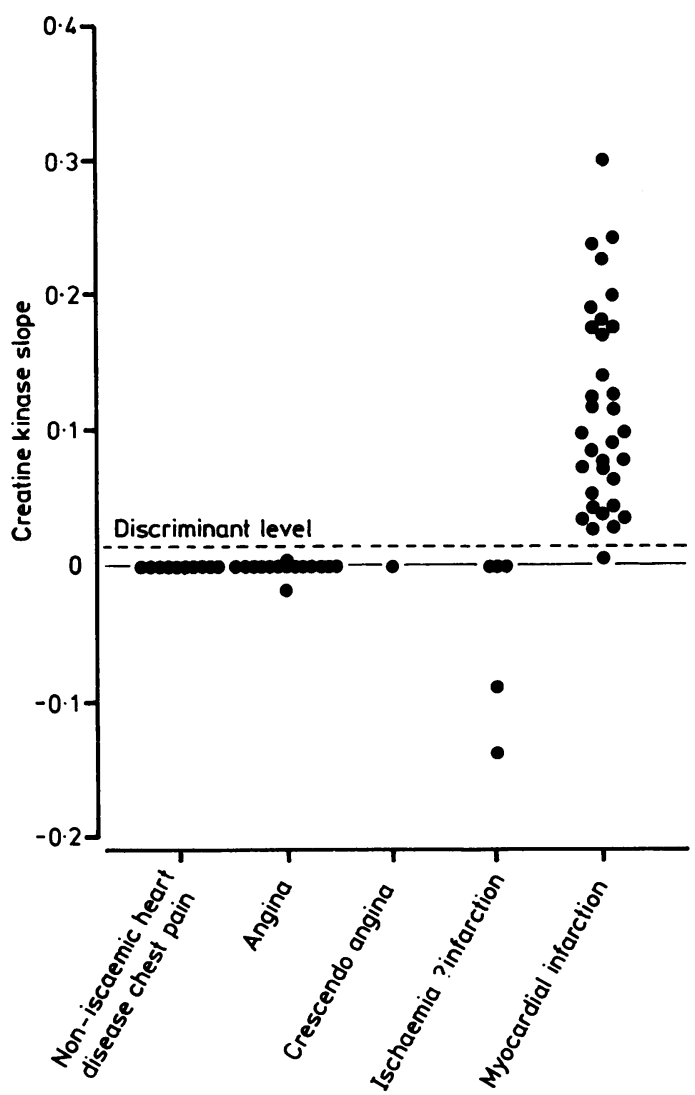

Fig 2 Change in log CK-MB per hour (laboratory determinations) using final diagnostic classification in patients with chest pain of 12 hours or less.

agreed with the final diagnosis in all cases of infarction, with clear separation between the infarct and non infarct groups (fig 1). Two of the patients in the ischaemia ?infarction group showed CK slope values within the infarct range.

Classification using CK-MB slope values showed almost complete discrimination of the infarct group from the other diagnostic categories (fig 2). CK-MB was not detected in any of the patients who had not had an infarct. One patient in the infarct group failed to show an abnormal slope value. CK-MB values in this patient were eight times the upper reference limit but showed a peak value in the second sample.

\section{DIAGNOSIS BY CK SLOPE (SERALYSER DETERMINATION)}

Determination of CK slope by the Seralyser showed good overall agreement with the laboratory values: Seralyser slope value $=0.92 \times$ laboratory slope, $\mathrm{r}=0.97$ (Deming regression).
Intrabatch imprecision for the Seralyser was $4 \%$ compared with $0.9 \%$ for laboratory analyses. CK slope results measured by Seralyser showed a similar pattern to those obtained using laboratory data. One of the patients with myocardial infarction and one with ischaemia ?infarction, with previously increased slope by laboratory determination, showed a Seralyser slope of less than 0.015. A cut-off level of 0.012 produced discrimination between groups virtually identical with that obtained using CK slope measured by the laboratory (fig 3 ). The difference between the two methods was due the effect of assay imprecision on the derivation of CK slope and is discussed more fully below.

Classification using the upper reference limits rather than the slopes for CK produced false positive results in three and false negative results in two. For CK-MB there was a false negative result in one patient and false positive results in two patients. These results are summarised in table 1.

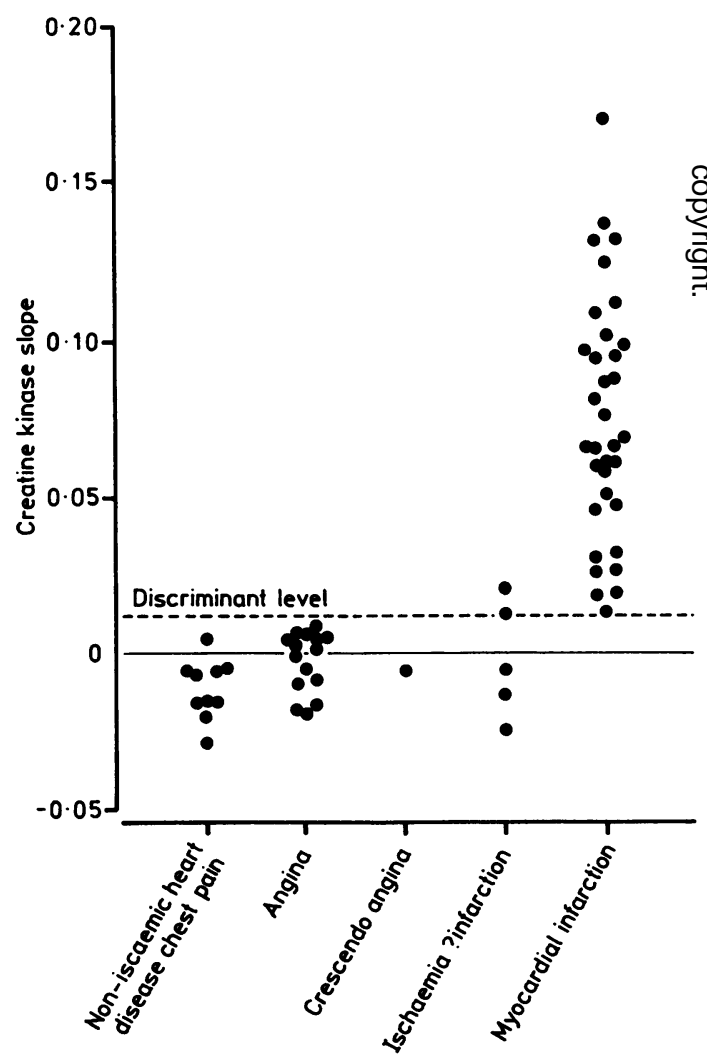

Fig 3 Change in log CK per hour (Seralyser determinations) using final diagnostic classification in patients with chest pain of 12 hours or less. 
Table 1 Positive diagnosis of myocardial infarction using only upper reference limits of $C K$ and $C K-M B(n=65)$

\begin{tabular}{|c|c|c|}
\hline Time & $C K$ & $C K-M B$ \\
\hline \multicolumn{3}{|c|}{ Non-ischaemic chest pain $(n=10)$ : } \\
\hline 0 & 1 & 0 \\
\hline 4 & 1 & 0 \\
\hline 8 & 0 & 0 \\
\hline 12 & 0 & 0 \\
\hline \multicolumn{3}{|c|}{ Angina $(n=15)$ : } \\
\hline 0 & 1 & 1 \\
\hline 4 & 1 & 1 \\
\hline 8 & 1 & 1 \\
\hline 12 & 1 & 1 \\
\hline \multicolumn{3}{|c|}{ Crescendo angina $(n=1)$ : } \\
\hline 0 & 0 & 0 \\
\hline 4 & 0 & 0 \\
\hline 8 & 0 & 0 \\
\hline 12 & 0 & 0 \\
\hline \multicolumn{3}{|c|}{ Ischaemia ?infarction group $(n=5)$ : } \\
\hline 0 & i & 1 \\
\hline 4 & $i$ & 0 \\
\hline 8 & i & 0 \\
\hline 12 & $i$ & 0 \\
\hline \multicolumn{3}{|c|}{ Infarct group $(n=34)$} \\
\hline 0 & 14 & 12 \\
\hline 4 & 27 & 29 \\
\hline 8 & 32 & 32 \\
\hline 12 & 32 & 33 \\
\hline
\end{tabular}

\section{Discussion}

Measurement of CK on admission and at four, eight, and 12 hours thereafter as used here provides rapid accurate diagnosis in patients admitted with acute chest pain. The diagnostic accuracy was matched only by patient classification using all available information.

Sequential blood sampling during the period immediately after infarct for diagnosis of acute myocardial infarction has been suggested previously. In the NORDKEM project ${ }^{8} 481$ patients admitted to hospital with chest pain and a working diagnosis of acute myocardial infarction were examined. This series showed that a total CK within the reference range in samples taken at 10 and 16 hours had a negative predictive value for infarction of 0.99 . In patients with increased total $\mathrm{CK}$, measurement of CK-MB confirmed the diagnosis of acute myocardial infarction with a positive predictive value of 0.99 . Bernstein et al proposed that a rise in CK-MB and $\% \mathrm{CK}-\mathrm{MB}^{9}$ or predicted peak CK-MB ${ }^{10}$ in samples obtained on admission and at six and 12 hours can be used for diagnosis. They observed that failure of CK-MB to increase at six hours can be used to exclude myocardial infarction, but we observed one false negative result in a sample taken at eight hours after admission. As shown here, however, rate of rise of CK alone (CK slope) can be used for diagnosis.

Measurement of CK-MB rather than measurement of $\mathrm{CK}$ is advocated on the grounds of specificity. We found that CK-MB slope failed to reach the discriminant level in one case of myocardial infarction because the peak CK-MB value occurred before completion of sampling. This is a reflection of the narrow time window for rise in CK-MB, a criticism of the use of CK-MB for diagnosis of acute myocardial infarction made by other workers." "Two patients in the ischaemia ?infarction group had a positive CK slope diagnosis unconfirmed by a CK-MB slope diagnosis. One patient presented with acute left ventricular failure and was clinically suspected to have had acute myocardial infarction. This patient subsequently had a cardiac arrest and died while in hospital. The other patient showed septal akinesia on echocardiography and had residual $\mathrm{T}$ wave changes. The $\mathrm{CK}$ changes in these patients may represent severe ischaemia alone, but failure to detect CK-MB may have been due to inadequacy of immunoinhibition as an analytical method as in both cases the CK values were low and within the reference interval. In our previous study no problems were seen at comparable levels of total CK, but sensitive, specific methods were used.

The data in our study did not show any improvement in diagnostic performance of CK-MB slope over total CK slope in patients with definite infarction. Release of CK-MM from damaged skeletal muscle might mimic myocardial infarction. This could cause problems in patients presenting with chest pain following muscle trauma such as intramuscular injection or operation if CK slope alone is used. This did not apply to our patients. In all but two cases the magnitude of the CK-MB slope was equal to or exceeded that of the total CK slope. This would probably not be the case in muscle damage where predominantly CK-MM is released. It would be expected that the value for total CK slope would exceed considerably the value for CK-MB slope: hence measurement of CK-MB slope would establish the diagnosis if this was a potential diagnostic problem.

On the basis of our findings the optimal diagnostic strategy would be to use CK slope for the patient presenting with chest pain, with measurement of CK-MB if an alternative source of CK is suspected (fig 4). The results obtained with determination of CK using the Seralyser on the coronary care unit were the same as the laboratory results. Hence measurement of CK could be carried out on the coronary care unit, leading to early decisions on treatment or discharge. Prompt diagnosis is helped by a suitable program to calculate slope. We have developed such a program for use on a microcomputer or programmable calculator. This could be integrated with a laboratory computer system to produce an automated interpretive report.

False positive and false negative results were obtained when the diagnosis was made using reference limits. Analysis of the data showed that CK slope had 


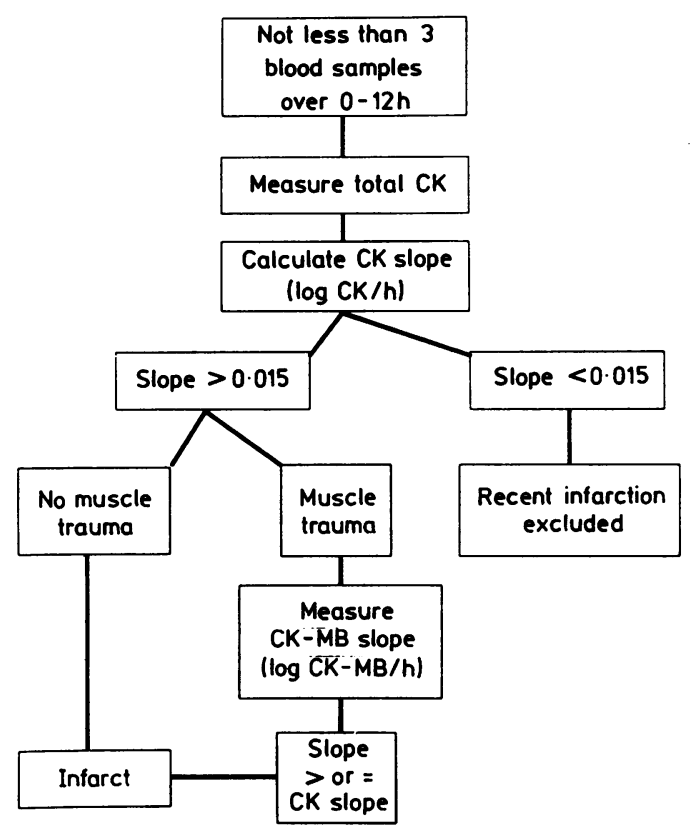

Fig 4 Diagnostic strategy

a sensitivity of $100 \%$ with a specificity of $94 \%$ compared with a sensitivity of $94 \%$ and specificity of $90 \%$ for diagnosis using upper reference limits for CK (table 2). A false negative result can occur as a consequence of changes in enzyme activity within the reference interval. This arises due to the magnitude of the reference interval for enzymes which is high compared with other analytes. Measurement of rate of change in CK slope uses the patient as his or her own normal control, which overcomes this problem. The technique is limited only by intrabatch analytical imprecision and biological variation over the period of sampling. From our data we would estimate median variation among patients as $11.7 \%$, yielding a maximum value for CK slope of 0.008 over 12 hours, although the maximum value we observed was 0.0055 (combined non-ischaemic chest pain and angina $\overrightarrow{0}$ groups). It can be calculated that if sensitivity of the $\stackrel{\vec{*}}{\vec{\sigma}}$ procedure is to be maintained every $1 \%$ increase in intrabatch imprecision will reduce the discriminant $\Rightarrow$ value by 0.0086 divided by the maximum time interval $\stackrel{\rho}{+}$ between first and last samples. This would explain the lower value of CK slope for the Seralyser determinations, as for the sampling times used, a slope value of $\frac{\sigma}{\sigma}$ 0.012 would be compatible with an assay imprecision $\stackrel{\varnothing}{\varrho}$ of $8 \%$ for the Seralyser. This probably reflects the true $\%$ value on routine use on the coronary care unit $\overrightarrow{0}$ compared with assay of control sera. We estimate that a slope of 0.01 would yield complete separation and set $\vec{\omega}$ minimum values for intrabatch coefficient of variation 9 (CV) and maximum duration of sampling, respec- 웅 tively, to $15.9 \%$ for 12 hours, $10 \cdot 1 \%$ for eight hours, $\stackrel{\vec{N}}{\mathrm{~N}}$ and $4.8 \%$ for four hours.

Other markers such as myoglobin, ${ }^{1213}$ cardiac specific lactate dehydrogenase $\mathrm{e}^{14}$ or subbands $\overrightarrow{\vec{N}}$ (isoforms) ${ }^{1516}$ of the MM isoenzyme of CK have been $\widetilde{N}$ proposed as more rapid diagnostic tests for acute $ᄋ$ myocardial infarction. None was comparable with CK $\overrightarrow{ }$ slope for speed or convenience and accuracy of $z$ diagnosis. $^{7}$

Confirmation of suspected infarction by measurement of the "standard" enzymes on consecutive days inevitably results in delay in diagnosis $\vec{\circ}$ and cannot influence initial patient management. ${ }^{17}$ I 8 contrast, CK slope produces results by 12 hours from. time of admission. This has major implications in vie of the recent findings that thrombolytic treatment is beneficial up to 24 hours from admission, ${ }^{18}$ although it yields better results the earlier it is administered. CK slope technique could be used for rapid confirmation $\stackrel{\square}{\triangle}$ or exclusion of acute myocardial infarction in patients $\overrightarrow{\vec{B}}$ in whom the diagnosis is in doubt before thrombolysis. $\frac{9}{3}$ This compares with the three days taken by the "standard" enzymes. Fifty four per cent of the admissions here occurred on a Thursday, Friday, or Saturday when there would inevitably be a further ? delay before the results of "standard" enzymes would be available.

Twenty five patients did not have acute myocardial

Table 2 Diagnostic performance of $C K$ and $C K-M B$ using upper reference limit (URL) at 12 hours from admission and using 윽 slope value

\begin{tabular}{|c|c|c|c|c|c|c|c|c|}
\hline & \multicolumn{4}{|c|}{ Using URL at 12 hours } & \multicolumn{4}{|c|}{ Using slope value } \\
\hline & \multicolumn{2}{|l|}{$C K$} & \multicolumn{2}{|l|}{$C K-M B$} & \multicolumn{2}{|l|}{$C K$} & \multicolumn{2}{|l|}{$C K-M B$} \\
\hline & Positive & Negative & Positive & Negative & Positive & Negative & Positive & Negative \\
\hline $\begin{array}{l}\text { Infarct } \\
\text { Non-infarct }\end{array}$ & $\begin{array}{r}32 \\
3\end{array}$ & $\begin{array}{r}2 \\
28\end{array}$ & $\begin{array}{r}33 \\
2\end{array}$ & $\begin{array}{r}1 \\
29\end{array}$ & $\begin{array}{r}34 \\
2\end{array}$ & $\begin{array}{r}0 \\
29\end{array}$ & $\begin{array}{r}33 \\
0\end{array}$ & 31 \\
\hline $\begin{array}{l}\text { Sensitivity } \\
\text { Specificity } \\
\text { Efficiency }\end{array}$ & \multicolumn{2}{|c|}{$\begin{array}{l}94 \% \\
90 \% \\
92 \%\end{array}$} & \multicolumn{2}{|c|}{$\begin{array}{l}97 \% \\
94 \% \\
95 \%\end{array}$} & \multicolumn{2}{|c|}{$\begin{array}{r}100 \% \\
94 \% \\
97 \%\end{array}$} & \multicolumn{2}{|c|}{$\begin{array}{r}97 \% \\
100 \% \\
98 \%\end{array}$} \\
\hline
\end{tabular}


infarction and could have been transferred from the coronary care unit earlier if the results of biochemical confirmation had been available. This would represent a $50 \%$ cost saving on the basis of transfer to a general medical bed alone. If early discharge from hospital occurs this saving would be higher. This would certainly be the case for the non-ischaemic chest pain and angina groups. On the basis of current bed cost within our hospital we estimate that there would be a potential annual saving of $£ 30,000$ for early transfer and $£ 75,000$ for early discharge.

CK slope would allow those patients with crescendo angina who have not infarcted to be prospectively identified, thus permitting early intervention by angioplasty or bypass grafting. This group of patients would benefit from further study.

None of the patients we studied had received thrombolytic treatment. Successful treatment with reperfusion is accompanied by earlier increase of CK and CK-MB, frequently with increased activity. Caution should be exercised in applying our proposed strategy in such patients.

In conclusion, timed sequential measurement of creatine kinase in samples obtained within 12 hours of admission has potential for very early, accurate diagnosis of myocardial infarction. A minimum of three samples obtained within this period, combined with calculation of CK enzyme and isoenzyme slope values, was superior to any other single diagnostic technique and better than the usual cardiac enzyme protocol. It permits very rapid biochemical confirmation or exclusion of myocardial infarction, with the opportunity for the laboratory to contribute to immediate patient management. It could also substantially reduce costs.

\section{References}

1 McQueen M, Holder D, El-Maraghi N. Assessment of the accuracy of serial electrocardiography in the diagnosis of acute myocardial infarction. Am Heart J 1983;105:258-61.

2 Gerhardt W, Waldenstrom J, Horder M, et al. Creatine kinase and creatine kinase B-subunit activity in serum in cases of suspected myocardial infarction. Clin Chem 1982;28:277-83.

3 Lee TH, Weisberg MC, Cook EF, et al. Evaluation of creatine kinase and creatine kinase-MB for diagnosing myocardial infarction. Arch Intern Med 1987;147:115-21.
4 Goldman L, Cook FE, Brand DA, et al. A computer protocol to predict myocardial infarction in emergency department patients with chest pain. $N$ Engl J Med 1988;318:797-803.

5 Collinson PO, Rosalki SB, Flather M, Wolman R, Evans T. Early diagnosis of myocardial infarction by timed sequential enzyme measurements. Ann Clin Biochem 1988;25:376-82.

6 Rosalki SB. Association of Clinical Biochemists Working Party Recommendations: proposed methods for determination of some enzymes in blood serum. Association of Clinical Biochemists News Sheet 1980;Supplement:9-12.

7 Ramahamdamy EM, Collinson PO, Evans DH, Fin RS, Baird IM. Evaluation of the Ames Seralyser for determination of CK on the coronary care unit. Clin Chem 1988;34:1914.

8 Committee on Enzymes of the Scandinavian Society for clinical chemistry and clinical physiology. Report on creatine kinase and creatine kinase B subunit activity in serum in suspect myocardial infarction. Helsinki: Finland. The Nordic Clinical Chemistry Project (NORDKEM), 1981.

9 Bernstein LH, Reynoso G. Creatine kinase B-subunit activity in serum in cases of suspected myocardial infarction: a predictive model based on the slope of MB increase and percentage CKMB activity. Clin Chem 1983;29:590-2.

10 Bernstein LH, Sachs E, Jonas A. Timed sequential measurements of creatine kinase MB in diagnosis of myocardial infarction. Clin Chem 1984;30:1588-9.

11 Griffiths PD. CK-MB: a valuable test? Ann Clin Biochem 1986;23:238-42.

12 Mainard F, Massoubre B, Marec HL, Madec Y. Study of a myoglobin test in patients hospitalised for suspected myocardial infarction. Clin Chem Acta 1985;153:1-8.

13 Chapelle J, Heusghem C. Semi-quantitative estimation of serum myoglobin by a rapid latex agglutination method: an emergency screening test for acute myocardial infarction. Clin Chim Acta 1985;145:143-50.

14 Bernstein LH, Scinto P. Two methods compared for measuring LD1/Total LD activity in serum. Clin Chem 1986;32:792-6.

15 Jaffe AS, Serota H, Grace A, Sobel BE. Diagnostic changes in plasma creatine kinase isoforms early after onset of acute myocardial infarction. Circulation 1986;74:105-9.

16 Wu AHB, Gornet TG, Wu VH, Brockie RE, Nshikawa A. Early diagnosis of acute myocardial infarction by rapid analysis of creatine kinase isoenzyme-3 (CK-MM) sub-types. Clin Chem 1987;33:358-62.

17 Anonymous. Diagnosis of the doubtful coronary. Lancet 1982;i:661-2.

18 Randomised trial of intravenous streptokinase, oral asprin, both, or neither among 17187 cases of suspected acute myocardial infarction: ISIS-2. ISIS-2 (Second international study of infarct survival) collaborative group. Lancet 1988;ii:349-60.

Requests for reprints to: Dr P O Collinson, Department of Clinical Biochemistry, West Middlesex University Hospital, Isleworth, Middlesex TW7 6AF, England. 